Voix et Images

voixetimages

\title{
La Belle Bête ou la double scène
}

\section{Béatrice Slama}

Volume 8, numéro 2, hiver 1983

Marie-Claire Blais

URI : https://id.erudit.org/iderudit/200381ar

DOI : https://doi.org/10.7202/200381ar

Aller au sommaire du numéro

\section{Éditeur(s)}

Les Presses de l’Université du Québec

\section{ISSN}

0318-9201 (imprimé)

1705-933X (numérique)

Découvrir la revue

\section{Citer cet article}

Slama, B. (1983). La Belle Bête ou la double scène. Voix et Images, 8(2), 211-228. https://doi.org/10.7202/200381ar d'utilisation que vous pouvez consulter en ligne.

https://apropos.erudit.org/fr/usagers/politique-dutilisation/ 


\title{
La Belle Bête ou la double scène
}

\author{
par Béatrice Slama, Université de Paris VIII-Vincennes
}

La sociologie de la littérature pose-t-elle les questions, propose-t-elle une méthode d'approche, des outils conceptuels qui permettent de aliren des romans de fermmes, dans leur spécificité?

«Romans de femmes "? La pertinence de ce découpage fait certes ellemême problème. La sociologie de la littérature peut-elle apporter des éléments de réponse, contribuer à la définition théorique d'une telle «spécificité »?

Les fondateurs de la sociologie de la littérature ne se sont guère posé ce problème et rares sont les textes de femmes qui ont retenu leur attention. Ainsi deux romans de Marie-Claire Blais sont-ils les seuls auxquels Goldmann ait consacré une étude.' II serait facile de montrer, en s'en tenant aux concepts de Lukàcs et Goldmann, à quel point les exigences esthétiques de "cohérencen, de "totalitén, de *question raisonnable "conviennent mal aux textes de femmes. Le statut social des femmes a été celui de l'enfermement, de l'exclusion, du partiel, du parcellisé : la contradiction interne, l'état de conflit jamais tout a fait résolu tissent leur écriture et les questions qui surgissent de tant de textes - qui suis-je femme? qu'est-ce que je veux? qu'attend-on d'une femme? comment exister, comment exister autrement? comment dire ce qui n'a pas été dit? - n'entrent sans doute pas dans celles que Lukacs trouvait araisonnables».

Mais pour les sociologues de la littérature, le fait littéraire est \& un fait significatif, une réponse d'un sujet individuel ou collectif, constituant une tentative en vue de modifier une situation donnée dans un sens favorable aux aspirations de ce sujet $x .^{2}$

Or le sexe s'inscrit dans la société comme une différence biologique mais aussi sociale et politique puisque la société a fondé sur cette différence biologique une hiérarchie, un rôle, un statut, un mode d'existence, un a territoire». A des situations et des problemes spécifiques, les femmes ont tenté et tentent de donner des réponses que nous avons justement à interroger. Comme elles ont eu. elles ont à percevoir et affronter a autrement* les problèmes globaux de la société. C'est à partir de ces différences que se fonde. selon nous, le présupposé, comme hypothèse de recherche pour une sociologie de la littérature. d'une spécificité elle-même relative et à étudier dans son historicité, des textes de femmes. 
Telle que Lukacs et Goldmann l'ont orientée, la sociologie de la littérature romanesque vise, dans la mise en relation. d'un texte et d'un “ réel $\mathrm{m}$, la a totalité * sociale: en falt, la structure socio-économique du capitalisme, son évolution. les étapes de la "réification». Mais ni Goldmann, ni Lukács n'ont pris en compte la totalité sociale qu'ils considèrent, la coexistence d'une autre structure socioéconomique. la structure patriarcale qui survit et se transforme au sein de la socıété capitaliste, dans un processus de soutien et de contradiction. Ses effets en sont manifestes non seulement dans la famille mais dans l'ensemble de la société. Elle institue des rapports fondés sur la hiérarchie des sexes. sur l'exploitation de la femme par l'homme, sur ce pouvoir que des femmes ont appelé, au XIXème siècle, le amasculinisme» et de nos jours la «phallocratie». La structure patriarcale pèse essentiellement sur les femmes. Assignée à une place où elle est définie, nommée, représentée, la femme est aussi chargée de légitimer et de reproduire, en tant que mère, le consentement féminın à la domination masculine. La structure patriarcale n'évolue pas au même rythme que la structure capitaliste. Plus que les hommes, les femmes vivent à la fois, dans le partage, l'antagonisme, le mal être, des temps historiques différents et des mentalités conflictuelles.

Si on ne tient pas compte de cette double structure et de ses effets. on passe à côté d'une dimension essentielle du socıal. On occulte les femmes. On méconnaît l'une des fonctions du roman qui est le retour du refoulé. ce que l'on appelle significativement «le privé», sur la scène du social et du politique.

C'est une banalité de rappeler que les femmes ont été prises dans un circuit d'échange et leurs corps réduits en marchandises qui circulent entre les hommes. Une sociologie qui met en relation le genre romanesque et le concept de valeur d'échange devrait s'intéresser à ce qui peut inscrire, dans des romans de fernmes, le point de vue des dominées, le point de vue des "marchandises".

Certes une analyse de la domination masculine renverrait à la complexité des rapports hommes-femmes, des rapports mères-filles et à leur enracinement dans les couches les plus archaíques, encore mal explorées, de l'imaginaire social. C'est encore là une dimension que ne peut ignorer une sociologie de la littérature.

Une lecture du point de vue des femmes me paraît nécessaire à intégrer dans une sociologie de la littérature, lecture attentive à ce qui surgit des textes de femmes, de l'intérieur: questions et réponses mal entendues ou occultées, dialogues dans et avec les discours dominants, figures de l'imaginaire et de la socialité, émergence d'une femme nouvelle, conflit entre l'ancienne ot la « nouvelle».

Je ne suis pas sans ignorer les objections que peut susciter une telle perspective. Et d'abord celles que ne cessent de susciter les mots a femme», a fémınin *. Piégés, connotés dans le discours social, ils risquent de renvoyer à je ne sais quelle "essence», quelle «nature de femme ou à l'opposition du " masculin» et du "féminin» figée en stéréotypes et rôles. Cette exigence est en quelque sorte imposée aux femmes par ce qu'Althusser, reprenant la 
parabole de la courbure et du bâton, a désigné comme la nécessité de la contrecourbure: con est bien obligé, puisqu'il faut forcer les idées à changer. de reconnaitre la force qui les maintient en état de courbure. en leur imposant une contre-force qui annule la première, la contre-courbure qu'il faut pour les redresser ${ }^{3}{ }^{3}$

Ce changement de point de vue, nécessaire pour lire les romans de femmes, devrait permettre de lire « autrement $n$ les textes écrits par des femmes.

\section{La Belle Bête lue par Goldmann}

L'un des romans étudiés par Goldmann en 1969 est la Belle Bête. ${ }^{4}$ Rappelons rapidement les grandes lignes de I"histoire narrée.

Trois personnages: Louise la mère, veuve et riche propriétaire terrienne. Isabelle-Marie la fille et Patrice le fils, "l'idiot" que sa scur a surnommé ala belle bêten, apparaissent à l'ouverture du texte dans un train qui les ramène de la ville. D'emblée, les rapports sont posés: Louise et Patrice sont blottis l'un contre l'autre, Patrice “la tête sur l'épaule» de sa mère - l'un des signifiants insistants du texte -; Isabelle en face d'eux, distante. Patrice est beau, admiré de tous, aimé de sa mère ; Isabelle est laide, mal aimée, haineuse et a révoltée». Dans ce trio familial menacé par le désir obsédant d'Isabelle de détruire la beauté de Patrice, deux personnages vont intervenir. Le premier. Lanz, l'amant de Louise devient son mari et s'installe a la ferme. Les liens qui unissent Louise et Patrice se distendent. La jalousie de Patrice, attisée par Isabelle. le pousse à tuer Lanz. Le second est un jeune aveugle, Michaël, qu'isabelle rencontre a un bal dans une ferme. Elle lui fait croire qu'elle est belle. noue une idylle avec lui et l'épouse. Elle devient mère mais Michaël recouvre la vue, découvre la laideur d'isabelle et son mensonge, la frappe et l'abandonne avec leur fille. Isabelle retourne dans le huis-clos familial et le drame se précipite. Isabelle plonge la tête de son frère dans une bassine d'eau bouillante. Patrice défiguré est abandonné par sa mère dans un asile où il joue avec un jeune fou, Faust. Louise chasse Isabelle et la petite Anne. Elle reste seule, rongée par un cancer de la face. Le livre s'achève sur la destruction des terres auxquelles Isabelle met le feu, et sur une triple mort: celle de Louise consumée par l'incendie, celle d'Isabelle qui se jette sous le train, celle de Patrice qui se noie dans le lac où il recherche sa beauté.

Goldmann nous donne de ce texte une brève lecture explicative. Dans I'univers sans issue de la Belle Bête. Goldmann voit ala transposition presque rigoureuse de la vision par de jeunes intellectuels a révoltés m mais aimpuissants" d'une *communauté canadienne-française", a société essentiellement agricole*, a spirituellement et intellectuellement sous l'influence et la direction de l'Eglise catholique, dont le caractère conservateur s'opposait au progrès et à la modernisation» et qui prônait ale mépris de la villen. De cette communauté "mortellement malade et condamnée», Louise, progressivement “ mangée » par le cancer, occuperait homologiquement la place.'

La démarche de Goldmann est fondée sur une homologie de structures qu'il pense découvrir en mettant en relation une vision du monde qu'il a 
préalablement analysée et ce qui lui paraît être la «structure significative» du roman. D'un côté, la a triade Patrice, Louise, Isabelle, homologue à l'ancienne communauté canadienne-française bête et ignorante (Patricel, à ses dirigeants spirituels qui, attachés à son caractère arriéré et agricole (les terres) la trouvent belle et l'admirent (Louise) et aux intellectuels de la nouvelle génération qui. encore impuissants, la haïssent et veulent la détruire (Isabelle)*; de l'autre, deux personnages, ales maris des deux femmes (p. 405). Pour Goldmann, a Lanz, le dandy venu de la ville et le blond Michaël ont dans le roman une place homologue à celle qu'occupent les Canadiens anglais dans la vision de cette société canadienne », "unis ou plus exactement mariés à la communauté canadienne-française traditionnelle dans l'État canadien, ce mariage sera détruit " par l'action des intellectuels canadiens-français de la nouvelle génération qui refusent la perpétuation de l'ancien état de choses * et a par le refus des intellectuels anglais de continuer l'union le jour où ils prennent conscience du vrai visage de cette nouvelle génération de Canadiens francais, de leurs exigences et de leurs aspirations» (p. 406).

Goldmann précise qu'il ne s'agit là que d'un a schéma global ø, a un point de départ diune analyse qui reste à faire» (p. 413). Mais, faute de « compréhension », «l'explication • semble plaquée de l'extérieur. Elle lie le texte sans vraiment le lire. Elle peut orienter un questionnement du roman. Elle ne peut permettre d'atteindre sa complexité et sa spécificité.

Mon propos n'est pas ici d'examiner la pertinence de cette piste de lecture proposée par Goldmann ni de critiquer tel concept de la sociologie goldmannienne. Mais parce que la démarche de Goldmann me parait ici exemplaire de ce refus de prendre en campte le aprivé * sinon comme al'homologue o d'autre chose et de cette inattention à un point de vue de femmes, à une voix de femme. je voudrais à mon tour lire la Belle Béte. Autrement.

Dans une première approche, je tenterai de montrer que, dans la perspective même d'une sociologie goldmannienne de la littérature, il était possible de ne pas s'enfermer dans cette lecture * unidimensionnelle* et de faire d'autres lectures de la Belle Béte. A condition de ne pas occulter - comme in-signifiant? - le «privé * dans le texte. A condition de ne pas occulter un «point de vue de femme». Et, pour tout dire, à condition de ne pas occulter le texte.

\section{Au-dela de l'homologie: le politique et le privé}

Différentes structures actantielles, pour ne s'en tenir qu'à elles, tissent dans le roman des réseaux signifiants multiples et hétérogènes qui coexistent, s'entrecroisent, interfèrent. De l'une à l'autre s'opèrent des glissements. Ambiguité des personnages, ambivalence du texte.

Une première structure est celle qu'a étudiée Goldmann. Il y a deux groupes de personnages dans le roman : le trio familial et "les autres $₫$. Étrangers à la famille. Lanz et Michaël correspondraient, selon Goldmann, aux Canadiens anglais, étrangers à la communauté canadienne-française. Leurs prénoms. 
opposés aux prénoms français de Louise, Isabelle, Patrice, pourraient, si nous voulions étayer l'hypothèse de Goldmann, en être lus comme signifiants. Mais l'explication que donne Goldmann de cette double union exogamique et de sa fin. pour séduisante qu'elle soit, ne suffit pas à rendre compte de la complexité et des modalités de leur inscription dans le texte. Elles renvoient, nous le verrons, à des rapports de couples et de sexes: Lanz et Michaël sont des personnages masculins, "autres" dans un univers féminin. Sur la double scène du sexuel et du politique. c'est l'impossibilité d'une communication réussie entre les sexes (les communautés?) et l'échec de la fusion dans le couple alors que les corps se confondent (dans l'État canadien? que pourrait signifier le mot "étrangers"), adormant l'un sur l'autre, ne se reconnaissant pas: amants étrangers 0 (p. 81).

Mais c'est par rapport au trio familial que se situent les * autres n personnages. Jamais adestinateur" toujours a adjuvant ou "opposant", adjuvant et opposant, al'autre - favorise le drame ou le réactive: c'est dans le trio familial qu'il se joue. L'opposition qui apparait comme l'axe sémantique du roman est celle qui déchire cette communauté familiale vouée à la fin du texte à la destruction. Peut-on, comme le fait Goldmann, n'y voir que la transposition des contradictions de la communauté canadienne-française? négliger ce qui est aveuglant? Le rapport de la fille a la mère explose tout au long du texte et nous y retrouvons l'écho d'autres romans de femmes.

Isabelle est l'exclue. Exclue du duo, en tiers. Sa haine du frère est aussi et surtout haine de la mère, de la mauvaise mère qui n'a «jamais su faire un juste partage entre ses deux enfants" (p. 156), qui n'a "jamais aimé sa fille, cette chair de sa chair \$ (p. 93). Ce rapport à la mère, conflictuel, dialogique est formulé dans cet étrange oxymoron du texte: a le bois brûlait en criant comme si la glace l'eût enfanté (p. 162). Appel et amour déçus, souffrance, haine, révolte, destruction. II est aussi identification. Les tentatives de vengeance contre Patrice allient curieusement la loi du talion et la figure maternelle. Traduisant le code affectif en code alimentaire. Isabelle prive Patrice de pain. comme sa mère l'a privée d'amour. Elle l'affame, mais elle le berce. Sa mère l'a « faite laide», elle × fera» laid Patrice. Elle brûle son visage mais elle lui caresse la nuque. Ma mère, mon miroir... Compulsion de répétition qui voue Isabelle à reproduire avec sa fille Anne dont elle ne supporte pas la laideur les rapports qui la lient a Louise. A la fin du texte, Isabelle regarde a la fenêtre Louise décharnée qui commence à lui aressembler *.

Dans son adoration même pour Patrice, Louise est encore une mauvaise mère: dans la beauté de son fils, c'est la réassurance narcissique de la sienne qu'elle recherche. Patrice défiguré, elle le rejettera avec horreur. Mère castratrice et aveugle qui se fait l'esclave de son fils. elle veut ignorer sa "stupidité * et l'aggrave: * Ce pauvre Patrice n'était pas né complètement idiot (...). Louise disait ce que l'enfant devait dire et jamais l'enfant $n$ 'éprouvait l'envie de chercher des mots». Progressivement, le statut de Patrice, le fils préféré, se modifie dans le texte. Tiers exclu et « révolté $n$ face à un couple. Louise et Lanz, puis Isabelle et Michaël, devenu laid et repoussé par sa mère il sera à la même place qu'lsabelle: il «partageait maintenant le sort fugitif d'isabelleMarie" (p. 172). 
Pour Goldmann, Louise est « homologue * aux "dirigeants spirituels « qui abêtıssent par leur éducatıon la communauté canadienne-française arriérée (Patrice). En somme, "la mère Louise serait la mère-Église: une figure de l'archétype de la "mauvaise mère». Interprétation qui n'a rien d'absurde. Elle pourrait même a expliquer * que Patrice ne puisse vivre que « la tête sur l'épaule * de cette mère tutélaire. Mais ne faire de cette femme, de ces rapports mère-fille, mère-fils. de ce conflit fraternel que de simples métonymies me paraît ne pas tenir compte de ce texte.

Une autre opposition se dessine dans le roman et clive autrement les personnages. Goldmann ne l'évoque pas. Elle s'offre cependant à une lecture goldmannienne. D'un cóté, Louise et Lanz, êtres inauthentiques, «mannequins», * marionnettes", "vieille poupée», "homme feint», qui vivent sur le mode du paraitre: cheveux teints, maquillage de Louise sans cesse refait, sans cesse réinscrit dans le texte : perruque et barbe fausse de Lanz qui tombent au moment de sa mort. lls sont attachés à la richesse, à « des passions vaines et artificielles ". De l'autre côté, Isabelle-Marie, Patrice et Michaèl, jeunes êtres ainstinctivement purs, a la recherche de leur *ăme*. Ce sont des êtres profondément doubles, victimes et coupables, violents et cruels parce que victimes.

\section{Cette opposition pourrait se lire sur deux axes.}

Ainsi il serait donc possible de retrouver dans la Belle Bête l'opposition si fréquente dans les romans québécois entre ville et campagne.

Louise et Lanz seraient du côté de la ville et de ce que Goldmann désigne dans la vision du monde qu'il analyse comme les valeurs de la ville : « égoïsme et matérialisme * correspondraient à une époque de modernisation et d'industrialisation et seraient al'apanage des Canadiens anglais protestants * (p. 402). Lanz le dandy vient de la ville. Louise, on ne sait d'où. Dans son premier mariage, elle a fait preuve d' $\alpha$ astuce calculée $\%$, elle a cherché $\propto$ à tout obtenir par son corps comme une prostituée, hantée par l'argent. (p. 30). Louise envisage des installations modernes. Son attachement à la terre est lui-mème intéressé. Ses a terres adorées * sont des terres d'or. Ses derniers mots seront, devant " ses granges debout comme des ventres d'or * : a je suis riche* (p. 208). Son mariage avec Lanz est l'union du même avec le même, «poupée mâle * et apoupée femelle* (p. 85).

Isabelle-Marie, Patrice et Michaël seraient du côté de la campagne,


ses terres comme des filles élues de Dieu» (p. 30). Isabelle a l'amour de la terre et du travail. Sa mère lui ôte tout en la chassant. Patrice erre à cheval en courses folles dans la forêt et se mire dans les lacs. Michaël appartient à la famille Livani - du côté de la vie - a famille immense, ferme comme un corps de glaise - (p. 110). Paysan et poète, il connait les bois, le vent, les saisons. l'histoire des récoltes. Tous trois se sentent tout proches des animaux.

La ville, absente dans le récit, semble signifiée par le train qui apparaît dès l'ouverture du roman. Train maléfique où Isabelle est seule avec sa haine. train qui ramène Louıse et Lanz, qui éloigne Patrice vers l'asile, qui exile 
Isabelle et Anne, train qui arrive au dénouement comme le destin: a je n'aime pas les gares», disait Isabelle. La campagne a des signifiants plus ambigus: la terre. bien sûr, blé et or, "terre de Louise " et "terre de Dieu», mais aussi le pain qui revient en leitmotiv dans le texte. Aucune autre nourriture n'est nommée. L'heure des repas, c'est l'heure du partage rituel du pain. Du pain et du vin. Après le père, le «maitre du pain», c'est à Isabelle que revient ce pouvoir. C'est elle qui cuit le pain, le rompt, le distribue. C'est elle qui le refuse. Quand Isabelle tient le couteau du pain, Louise tremble comme devant une menace. Pain sacré, pain de la haine, signifiant double comme sont doubles les personnages, du côté de la campagne.

Le texte condamne les valeurs de la vilie. II inscrit aussi le processus de dégradation de la vie à la campagne. Il dit le drame de la destruction de la terre comme demeure. C'est un roman de la dépossession que signifie, dans l'intertextualité. l'exergue de la seconde partie, emprunté au livre d'lsaïe:

Ma demeure est enlevée et emportée Comme une tente de bergers.

La petite Anne est abandonnée symboliquement sur le quai de la gare. alors que les terres flambent, elle aqui n'avait même plus envie de partir. de jouer, ni même de vivre * (p. 160). Aucune issue pour elle ni à la campagne ni à la ville.

L'axe des générations permet une lecture à un autre niveau. Le point de vue des anfants (p. 100) condamne la corruption des adultes. des a époux fanés* (p. 73). Louise et Lanz dont la chair même se corrompt. La prolifération dans le texte des signifiants du processus de corruption du corps de Louise est obsessionnelle: de la anervure au «sillon», au "lierre effrayant * a la - crevasse *, la « lèpre *, au * ver qui mange sa joue *, au * destin de putréfaction *, au "cadavre a la fenêtre" qui * nage dans le pus». Lanz appartient à ce mème monde qui an'a jamais eu de jeunessen, ce "monde en décomposition " de Louise. Tout pourrit et se défait au moment de sa mort. Condamnation sans merci au niveau de l'énonciation.

Mais les xenfants» ne sont pas sauvés dans cet univers du román qui inscrit leur dualité. Faust, le comédien fou de l'asile et la petite Anne sont aussi, dans le système des personnages, du côté des pur-e-s, des mal aimé-e-s, des abandonné-e-s: les laids, les idiots, les fous, les aveugles. En marge du monde des adultes, ils s'évadent dans les jeux «pathétiques». « Jeux» de Michaël et d'lsabelle dans leur Paradis idyllique, afous de rires, assoupis dans les fleurs», rêveries et jeux sans fin de Patrice, théâtre de Faust. Jeux, pureté : signifiants insistants dans le texte. C'est leur face «de jour». Comme le met en abyme l'exergue de la première partie du roman sur les êtres doubles, ils ont aussi une face *de nuit", une face ad'épouvante». C'est l'obsession destructrice d'Isabelle, la violence de Patrice qui brise verres et miroirs, tue le chien d'Isabelle, précipite Lanz sous les sabots de son cheval, le cruauté perverse de Michaël qui s'acharne sur des insectes. frappe Isabelle, la pulsion déjà criminelle d'Anne qui suggère l'incendie à sa mère: si tu répandais la lampe, atout brûlerait» (p. 206). Violence qui les dresse contre les adultes. 
mais aussi les uns contre les autres et qu'ils retournent finalement contre eux-mêmes. Isabelle et Patrice se donnent la mort.

Ils sont monstrueux mais innocents parce qu'ils sont fondamentalement victimes. Comme Patrice, ils "cassent", afaute de ne pouvoir étreindre" (p. 68). Comme Faust, ils meurent, "au moment où la violence du drame le(s) consumait" (p. 199). Leur mort n'est pas décomposition, mais quête encore. Elle est donnée à lire dans une ellipse, un blanc. Ce qui est inscrit dans le texte, ce n'est pas la mort, mais la dernière aspiration d'Isabelle, son dernier regret, la marche vers le rail, une palpitation d'eftroi, c'est le passage du bleu du lac où Patrice cherche son «beau visage * au bleu du ciel où il retrouve son âme.

Cet univers où l'heure des repas, du partage du pain et du vin n'est pas celle de la communion, mais $\propto$ |'heure de nudité $*(p .75)$ × où les ennemis d'une mème famille se jugeaient silencieusement $\%$ où des adultes égoïstes et corrompus sont voués a la mort violente, où des a enfants " brûlés de désirs et d'aspirations frustrées sont acculés à la brutalité, au crime, au suicide, où les fous et les idiots "s'en vont toujours à la recherche de leur áme», où l'on ne peut «ni prier ni vivre, mais uniquement souffrir * (p. 199), cet univers de la violence est un univers sans la grâce. On est un peu surpris que l'auteur du Dieu caché n'ait pas fait cette lecture.

Isabelle se nomme aussi Marie. Marie comme la Vierge, mais elle est une avierge monstrueuse» (p. 110). Fille laide qui aspire peut-être à Dieu. elle est privée de la grâce, dis-graciée. Privation qui la brûle, la consume. Elle est atteinte au «olus sacré d'elle-même». Comme la sorcière de Michelet, elle est une vilaine habitée par le démon. Elle espère trouver l'amour et la beauté dans te mensonge. Faust féminin qui vendrait son âme au diable pour être belle? Rejetée définitivement dans le désespoir, le amal\%, elle est «démoniaque», «sorcière», «criminelle». Elle vit sa jalousie comme «une passion de damné: la satisfaire ou mourir* (p. 150). Devant la bassine d'eau bouillante, elle est «tentéen, «elle devait ressembler à Ėve préparant sa séduction (p. 163). Sa main est a comme une griffe». Elle est possédée, les pulsions perverses lui viennent comme d'un dedans étranger et son crime est un exorcisme: aaussitôt son geste sorti d'elle-même», Isabelle est «contentée $\times$ (p. 164).

Dans ce mande sans Dieu, sans âme, afétichiste», Louise s'est faite prêtresse du dieu Patrice, mais le vrai dieu de Louise, c'est son miroir et elle fera du miroir le dieu de Patrice. Au moment de mourir, elle a un cri: $\alpha$ Grand Dieu, pitié I*, x mais son miroir ne bougeait pas* (p. 212). C'est un univers de crime et de châtiment. Louise a méritait s son sort. Elle est livrée au *jugement dernier $n$, elle, la «prostituée», a cette mère qui n'était même pas une femme» (p. 208). Ce monde sans Dieu ne peut aller qu'à \& 'apocalypse *, dans les « vipères rouges * de l'incendie infernal (p. 212). Dans une optique chrétienne, sans doute pourrait-on voir la le terme d'un processus de décomposition et de damnation. l'arrivée de cette «fin des temps» qu'énonce l'écriture.

Univers romanesque à mettre en relation avec l'effondrement des valeurs chrétiennes dans une société en rupture avec les traditions ancestrales 
que symboliserait dans le texte le père absent? On pourrait aussi parler de résurgence d'un jansénisme que l'on retrouve ailleurs, dans la littérature québécoise.

A la fin du roman, dans l'acmé de la violence, après le double matricide - Isabelle a tué la terre et sa mère brûle dans l'incendie - il y a comme une conversion du personnage. Elle aspire à "avoir un dieu, un seul dieu», elle "aurait voulu donner autant qu'elle avait détruit" (p. 213). Conversion chrétienne?, "Conversion" du personnage problématique au dénouement du roman? Mais dans cet univers sans la grâce, il niy a pas de salut. Seul l'«idiot» parait sauvé. Le texte se ferme, dans la dernière phrase, sur ce bleu de ciel où Patrice retrouve son âme. Dernière note bleue dans un livre noir. Note d'espoir? Paradoxe qui signifierait aussi dans l'ambivalence le nom de Faust dans le roman: se perdre pour se trouver?

Autre structure actantielle: Isabelle-Marie, d'un côté, Louise, Patrice, Lanz, Michaël de.l'autre. Seule contre tous. Isabelle est le sujet d'une quête: vivre, exister. Une quête où elle est entourée d'opposants.

Isabelle-Marie - les signifiants de la femme dans l'imaginaire social occidental - est une femme. Une femme qui cherche a exister dans une société qui la nie et la condamne: «je veux vivre et respirer et voir malgré mon visages, ce visage qu'on lui a fait - ${ }^{\prime} N^{\prime} e s t-c e$ pas toi qui m'as faite si laide? - (p. 179). Femme en quête d'identité qui demande à être reconnue par le regard, la parole - «arle, mère - de la mère et des hommes. C'est d'eux qu'elle attend la reconnaissance de sa féminité. Mais elle est confrontée à une image - la beauté - qui lui est opposée comme une norme et la condamne puisqu'elle est laide. La laideur est vécue comme le mal qui met en question son être de femme, son être tout court. Car Isabelle est rejetée par le regard des autres. "Seul un aveugle pouvait la voir comme belle (p. 62). Isabelle va donc jouer à ètre belle. Et quand sa beauté - donc sa féminité est nommée, reconnue, füt-ce dans l'aveuglement, par Michaël, elle commence à devenir femme. * Isabelle naissait a l'amour de Michaël ; ; elle est aconvertie en vivante (p. 177). Mais Michaël, en cessant d'être aveugle, passe du côté des opposants: «il a eu des yeux pour me voir et je l'ai dégoûté» (p. 179).

Dans cette dialectique du regard et de l'être, lsabelle perd son être. Perte qu'inscrit le passage d'une thématique du fluide, de l'humide au sec: son àme est à $\times \sec \times$, a asséchée $*$, alors qu'elle était $\times$ fluide, assoiffée $\times(p .179$ ).

Si ce texte est l'histoire de la destruction par une femme, il est aussi I'histoire de la destruction d'une femme.

Si simplement Louise eût osé aimer sa fille. Naturelle, IsabelleMarie eùt grandi sans méchanceté. Elle était devenue cynique en répondant mal à la passion qui la sous-tendait. La perversité était chez elle une seconde nature comme ces êtres doubles qui ont une vie le jour, et une autre, plus effrayante, la nuit (p. 92).

Parce qu'elle n'est pas, parce qu'on la nie, il ne lui reste que le faire: le crime. Elle sait, elle veut, mais elle ne peut rien sinon détruire: *Tu me condamnes mais mon crime était ma seule façon de vivre» (p. 179). 
Le texte met en scène la collusion entre la mère et les hommes contre la fille-femme en quète d'identité. Collusion que dénonçait explicitement l'ouvrière Louise Tardif au Congrès ouvrier de Marseille en 1879. Ce qui peut paraitre un matriarcat dans le roman - le père est absent, la mère possède les terres, c'est chez elle que vient vivre Lanz - n'est qu'un pouvoir patriarcal détenu, usurpé mème dans l'énonciation, par la mère et exercé comme celui de ihomme contre la fille, contre la femme. Une perspective goldmannienne aurait pu établir une mise en relation avec l'image de la mère dans la société québécoise où celle-ci détient de l'Église une sorte de délégation de pouvoır et exerce, dans une absence relative de père. sa domination à l'intérieur de la famille.

Isabelle occupe dans le roman une position centrale. Non qu'elle focalise ni qu'élle en soit l'héroïne. L'univers de la Belle Bête est un univers sans a héros . Comme les autres personnages, elle est inscrite dans une énonciation ambigué, ambivalente qui la met à distance. l'accuse mais aussi l'excuse, lui donne la parole. Isabelle poursuit cette quête d'identité qui donne au roman sa cohérence comme son ambivalence et sa tonalité violente et dramatıque. Cohérence et ambivalence qui tiennent aussi au statut de Patrice dans le texte. Ambiguïté sexuelle de «la belle bête»: mi-enfant, mi-homme, il est énoncé-nié comme homme et désigné. par son surnom, du côté du. féminin. Ambiguïté de place dans le système des personnages: opposé à Isabelle dont il est involontairement le bourreau et la victime, il passe, on l'a vu, du même côté qu'Isabelle, à la même place qu'elle. Ambiguïté d'être double: «saint des légendes médiévales», « Saint-François» et afauve en a délire», Patrice apparait à l'analyse comme un double d'Isabelle. Derrière le conflit fraternel. se dessine un inquiétant déroulement des personnages et de la quête. Retrouver son «être», son "vrai visage», son «âme», la gràce, l'identité, l'enjeu est le même dans le texte. « Naître * ou « mourir * (p. 97). Quiètre? Comment ètre?

Le texte pose ces questions d'un point de vue de femme «révoltée», dans les conditions et les limites qui sont les siennes dans cette société et à son époque: celles du récit. Le texte donne a lire une femme. Des problèmes de femmes. L'ignorer, comme l'a fait Goldmann, c'est mettre à l'écart une dimension essentielle du roman. C'est en fait, une option idéologique.

Mais aussi ne pas lire l'écriture comme l'autre ascène où se libèrent des forces archaïques, où s'inscrivent le sexe et le désir, et n'y voir que la «transposition d'une "vision du monde - où le sexe. le désir, l'amour et la mort ne seraient que les signifiants des forces et des conflits en jeu sur l'échiquier social et politique, ou de simples *éléments antagonistes» dans le processus qui mène à la cohérence du texte - fait courir le risque non de découvrir un autre du texte mais de construire un autre texte. Construction qui serait aussi occultation.

\section{L'autre scène}

C'est sur cette "autre scène que je voudrais poursuivre ma lecture de la Belle Bète. ${ }^{5}$ 
L'univers de ce roman se tisse dans un travail de l'écriture qui combine étrangement et remodèle le conte de fées et le mythe, le thème romanesque et la parabole. Cendrillon et Narcisse. la Belle et la Bête, Oedipe. Electre, et "le père absent". Faust et le "miari aveugle". Formes culturelles qui renvoient à un imaginaire collectif, à des forces profondes, elles inscrivent dans un texte qui domine la violence, une inquiétante polysémie.

Cette société romanesque est une petite communauté familiale repliée sur elle-mème, comme coupée du reste du monde. Les seuls lieux de contact avec le "dehors" et les "autres" sont, jamais décrits. à peine nommés, le train, le bal, l'asile. L'essentiel du roman se déroule “dedans», dans la maison familiale, lieu indéterminé, espace clos qui s'ouvre sur les terres, les lacs, les bois. Lieux «quasi mythiques", dans un temps hors du temps social: seuls le train, l'évocation d'une modernisation des terres sont les indices d'une relative contemporanéité. Ce temps - une quinzaine d'années - est rythmé par les saisons, ales jours qui succèdent aux jours» (p. 142), et le retour symbolique de l'heure des repas. Les personnages n'ont pas de nom: des prénoms, un surnom, des épithètes. Indétermination et anonymat caractéristiques de l'univers des contes. Ici, les objets de la quête sont la beauté. l'amour, l'identité, l'âme.

On peut reconnaître dans Louise la figure de la marătre de Blanche-Neige qui interroge inlassablement son miroir et dans Isabelle celle de Cendrillon. Une Cendrillon vouée aux travaux de la terre, "le dos brûlé par le soleil* pendant que Patrice ajouait sa vie de fainéant» et que Louise avivait de caprices» (p. 20), une Cendrillon qui va au bal à la rencontre du Prince Charmant. Avec Patrice, on entre dans le mythe. On retrouve, étrangement fondues, la figure de "l'idiot», du asimple» mais aussi les grandes figures mythiques. Celle d'Oedipe: un Cedipe qui écrase sous les sabots de son cheval le substitut du père ( a je suis votre père”. p. 55 et 83 ), ce Lanz qui occupe le lit de la mère et, barrant l'accès de sa chambre, interdit la mère au fils. Celle d'Oreste: un Oreste qui, poussé par une Isabelle-Electre, que tue un LanzEgisthe. Celle de Narcisse: un Narcisse qui a a la folie des miroirs» (p. 123) et des eaux et descend au fond du lac pour y trouver son visage. Comme dans les contes de fées, la mort est ici transfigurée, par une euphémisation poussée jusqu'à l'antiphrase, en re-naissance. L'écriture joue avec ces " mythologèmes * jusqu'à la surcharge. Ainsi étrangement, Lanz est boiteux et Isabelle a de naissance une cheville plus mince que l'autre. C'est à la fois son intérêt et sa faiblesse.

Cette présence dans le roman du conte et du mythe n'est pas simple reprise, simple transposition. Elle est ré-écriture. Le texte reprend, inverse. déconstruit, parfois révèle le sens latent du conte ou le détourne. met en scène le mythe d'un autre point de vue. Réécriture de femme, pensons-nous. Même si elle ne s'inscrit guère dans ce que l'on a appelé, ces dernières années. une a écriture féminine".

Isabelle n'est pas servante. Le travail de la terre est son hâvre et son cilice. Elle est Cendrillon parce qu'elle est privée de l'amour de sa mère qui n'a de regard que pour le frère. Ainsi devient ici manifeste le sens du conte. 
tel que l'a analysé Bettelheım. Maıs Cendrillon est soumıse, résignée; Isabelle est révoltée, elle a "des rêveries aiguès et diaboliques". Cendrillon a une marraine: Isabelle n'a pas d'adjuvant, sinon la cécité de Michaël, sinon ce démon qui l'habite au bal. Le mensonge d'Isabelle apparait comme une ruse euphémisée en jeu. Mensonge, feinte, principale vertu des femmes selon Colette. Arme des faibles, des opprimés dans les contes; de ceux qui, ne pouvant compter que sur eux-mêmes, tentent d'agir pour réparer le manque. gagner une épreuve dans la poursuite de leur quête. Ce mensonge est aussi un don magique que se fait Isabelle. Mass à minuit, quand Isabelle-Cendrillon retrouve son apparence, le Prince-aux-cheveux-blancs, le a jeune dieu sauvage * l'abandonne. "Tu vois par miracle. Michaël, moi aussi c'est par miracle que j'étais belle. (p. 141). Mais il n'y a pas de miracle. L'amour heureux n'existe que dans les contes qui s'achèvent avant que ne commence la atragédie. L'amour ne vit que dans les «jeux», la fiction, l'imaginaire. Dans l'aveuglement.

C'est ce que dit encore le roman, en faisant des variations sur la figure du mari aveugle» qui, dans de nombreux textes de femmes, inscrit l'aspiration féminine à la fusion dans le couple. La femme devient les yeux, le bras, l'indispensable compagne de chaque instant. Celle aussi dont on ne verra pas la beauté se faner. Michaèl est le «frère-époux*, le «frère-enfant* (p. 133). Isabelle prend possession de son a infirmité (p. 75). Regard menaçant, toucher rassurant: c'est dans la fusion des corps et l'effusion des âmes que s'exalte l'union d'Isabelle et de Michaël. Mais le texte déconstruit le théme en opposant une « vérité romanesque "à un amensonge romantique»: l'amour est aveugle mais il cesse dès que cesse l'aveuglement.

Vérité désolée et pessimiste: celle de l'impossible amour heureux. Celle que chantent les poètes et que racontent les romans de femmes. Celle de l'impossible union politique, aurait dit Goldmann. II ne reste à cette Cendrillon qu'à tout réduire en cendres.

Le titre du roman joue sur la Belle et la Bête. Mais ici, la femme - traditionnellement la Belle qui rend à la Bête sa beauté - est laide. La Belle est aussi la Bête et lsabelle privera « la belle bête « de sa beauté, la métamorpnosant en une sorte d'animal monstrueux. Il y a là une inversion: inversion des sexes, inversion des rôles, inversion du message traditionnel du conte. « Sois belle et tais-toix, dit-on à la femme. Isabelle est laide et ne se tait pas. Patrice est beau et se tait. Si Patrice est la *bêtex - l'idiot -, c'est Isabelle qui serait la Bête du conte. intelligente, bonne - elle l'eût été si sa mère l'eùt aimée -. mais repoussante de laideur. II n'y a pas de Belle salvatrice: comme l'a fait la Belle Louise, le Beau Michaël se détournera d'Isabelle, "dégoûté».

Dans les contes, c'est l'harmonie des sexes et la métamorphose par l'amour dans une distribution traditionnelle des rôles. Ici, cette distribution est subvertie et les sexes se sépareront.

Louise évoque à la fois la marâtre de Cendrillon et la reine à son miroir. le bourreau de Blanche-Neige. Ce que le conte désigne comme la jalousie d'une femme supplantée dans sa beauté est manifestement dans le roman une rivalité entre la mère et la fille, un aduel» (p. 178) de femmes. Louise 
voudrait "forcer " Isabelle à se taire, mais c'est la mère qui est la plus belle, c'est la fille qui est jalouse et veut se venger. Louise, "la-belle-de-corps" (p. 30), admirée, “approuvée " par Lanz, Patrice et son miroir, s'épanouit, « repue” (p. 44). Isabelle la laide, abandonnée par Michaël, souffre d'une "immense déchirure de femme" (p. 141). Le corps de la mère, sa sexualité sont inscrits dans l'horreur et la fascination. Comme la méchante reine de Grimm, Louise s'évanouira dans le feu, "comme une danseuse à la fin d'un baltet" (p. 212). Mais ici, c'est la fille qui allume le feu et, comme elle l'annonçait à sa mère (p. 179), elle mourra elle-mème de cette jalousie.

Le meurtre de Lanz par Patrice est une mise en scène de l'Oedipe, c'est trop évident pour qu'on $y$ insiste. Mais ce qui paraît le plus intéressant dans le texte, c'est le travail sur les signifiants, les métonymies phalliques, de la canne d'or de Lanz - lance? - jetée sur le lit défait de la mère et que le fils découvre en sanglotant, au fouet de Patrice qui frappe "le grand corps mâle qui le narguait près de sa mère» (p. 83), au cheval qui écrase Lanz dans sa course folle. C'est surtout la présence, dans cet Oedipe, du a côté de Jocaste». Le texte tisse un climat d'intimité très étroite - incestueuse? - entre la mère et le fils: contact des corps, étreintes, moments privilégiés dans la chambre quand Louise défait ses cheveux. Lors de son départ à la ville, Louise, privée pour la première fois de Patrice, se sent "coupée en. deux» (p. 30). Contre Lanz qui la sépare de son fils, elle défend son amour mais elle $*$ ne sait plus parler a Patrice «rebelle» et vit dans la peur et l'angoisse de le perdre. Elle est « la torturée de son fils et veut retourner à « ce fils-bien-mal-aimé ( $p .120$ ). Louise comprend le meurtre de Lanz et lui donne sa signification: "Tu voulais être complètement mon fils» (p. 129). Elle le supplie de ne ajamais la quitter pour une épouse, une amien: aje suis ta mère, ta meilleure compagne. Je suis de toi et tu es de moi» (p. 160).

Intérêt du texte aussi: la place d'un Oedipe au féminin, le travail sur la figure d'Electre. L'attachement d'isabelle au père et aux valeurs du père, son identification au « maitre du pain * par la prise de possession des signifiants de cette maîtrise: le pain et le couteau sont a lire dans la sexualisation de l'inscription textuelle du père, $\alpha$ en labourant le ventre vierge de la terre, il pénétrait le caur de Dieu» (p. 57). Et Isabelle évoque le signifiant de cette puissance phallique, «les bottes énormes» qui sentent «le blé et la glaise* (p. 57) et que, Oedipe heureux, Patrice portera après la mort de Lanz (p. 149). Vêtue de noir, enfermée dans son a mal * et dans " le mépris de Louisen, Isabelle quittera fiancée sa tunique de deuil comme si l'amour de Michaël mettait fin à son veuvage.

Le *père absent» - que l'on retrouve souvent dans les contes de fées et dans les romans québécois - fonctionne dans la Belle Bête dans le rapport que son inscription institue avec les autres personnages. C'est parce que Louise l'a attiré dans ses filets et en a fait son «jouet \$ qu'elle apparait dans le texte comme la aprostituée et c'est parce qu'il prend sa place que Lanz fait figure d'Egisthe. Une autre lecture pourrait être proposée de la atriade familiale». Dans le couple Louise-Patrice. Patrice est l'enfant qui n'est pas encore séparé de sa mère, celui qui a "la tête sur l'épaulex, «dans le sein* 
de sa mère - jeu de signifiants: Patrice, la belle bête, un abé-bé × qui a tête * le "lait" dans le "sein" de sa mère? - l'enfant qui n'a pas accédé au stade du miroir ("Suis-je un miroir ou suis-je Patrice», p. 128), qui n'est que le reflet de sa mère et n'a pas d'âme parce que sa mère lui prète la sienne. Face a ce couple. Isabelle pourrait représenter symboliquement le père qui veut séparer le fils de la mère et le punit pour prendre sa place. En plongeant la tète de Patrice dans l'eau de feu. Isabelle casse le miroir. Patrice ne pourra plus être le reflet de sa mère. Coupé de Louise, il commencera à prendré conscience de ce qu'il est et de ce qu'elle est. La fin du texte est ambiguë: dans les eaux du lac où il retourne comme en un sein maternel, il ne se reconnaît pas et c'est derrière le miroir, de l'autre côté du miroir - comme il vivait déjà a à l'envers de son visage" (p. 174) - qu'il ira se chercher. Coupure définitive du "réel»? Et/ou possibilité enfin, après la mort de Louise, de xretrouver son âme?.

Cette interprétation de la fonction d'Isabelle permettrait de comprendre une phrase étrange du roman (p. 164). Après la destruction du visage de Patrice, «contentée. elle descendit le sombre escalier qui conduisait aux pièces closes depuis la mort de san père (le tombeau paternel?). Comme si. ayant accompli le devoir du père - la castration symbolique du fils - elle pouvait enfin «descendre» vers lui. Schéma initiatique? Identification au père qui expliquerait qu'elle n'accède pas réellement à la féminité? Isabelle, a le glaive ébréché * (p. 42), la mal castrée.

Ré-écriture du mythique, polyvalence des significations.

Comment lire la castration de «la belle bête», castrée par la mère de ses mots et de son àme. par la sceur-père, de la beauté et de l'amour de sa mère. comment lire la négation d'Isabelle que sa mère *n'a jamais osé regarder vraiment (p. 14), qui «n'a jamais eu un vrai visage d'enfant- (p. 12), atteinte «au plus sacré d'elle-même». la pureté (p. 78)? Comment lire le rejet de la laideur d'Isabelle, de Patrice? L'absence de tout équivalent de la puissance salvatrice des contes de fées, l'absence de toute perspective? Faut-il lire le rapport d'un fils-fille à une mère (société?) castratrice qui ne lui permet pas l'accès aux mots (au «symbolique * selon Lacan), l'accès à l'être ? le rapport d'une fille-femme a la mère et à l'homme qui la rejettent parce qu'elle ne se conforme pas aux normes - être belle et bête? Le texte ne répète-t-il pas inlassablement à travers Isabelle. Patrice, la petite Anne, la perte primordiale, l'abandon de la mère? Ne répète-t-il pas la "soif de protection», de sécurité, le besoin de la chaleur du contact charnel? Nostalgie de la *véritable mère* (p. 165), de celle qui reconnaîtrait son enfant et l'aimerait dans sa laideur même. Nostalgie de l'impossible. Louise est vue et ajugée × du côté de la fille. Mais Isabelle. mère à son tour, nous fait passer de l'autre côté, du côté de la difficulté d'être mère. Isabelle aime a malgré elle * Anne mais ne peut supporter sa laideur. Elle souhaite mourir et est apresque tentée de la tuer $>$ (p. 155). Rejet de la laideur et rejet de la mère se confondent: "Personne n'a eu pitié de moi puisque tu me repoussais * dit isabelle à sa mère (p. 179). Isabelle est * universellement sevrée» (p. 180). Patrice *au bout de l'abandon humain. (p. 173), Anne a froid comme, au début du texte, Isabelle avait la fièvre, mais 
nı l'une ni l'autre mère ne répond à l'appel de son enfant. "Castration" féminine? Déréliction d'une nouvelle génératıon abandonnée par ses dirigeants. sa communauté?

Ainsi pourrait-on lire dans la présence obsédante des mirorrs. des glaces. eaux. regards. miroir de Blanche-Neige et miroir de Narcisse, le narcissisme mortel de la mère qui ne permet pas à ses enfants d'exister et/ou celui de "la communauté canadienne-française" mais aussı la quète par Isabelle et Patrice d'un narcissisme réparateur pour tenter de trouver une identité.

A la fin du texte, Isabelle croyait "tuer la terre de Louise " et comprend qu'elle a "tué la terre de Dieu» - de son père? Si elle n'a plus d'issue dans sa quète d'identité. c'est qu'elle ne sait plus quelle est sa cible, c'est qu'elle s'est peut-ètre trompée de cible. En termes nietzschéens. elle s'est trompée de "dragon " et ne peut pas encore créer de valeurs nouvelles. D'ailleurs, en mentant à Michaèl, niest-elle pas entrée dans le jeu des valeurs qui la condamnent: la beauté comme signe de la féminité? Fin ambiguë, ambivalente qui traduit une association profonde et/ou le retour de la révoltée à une idéologie traditionnelle: aelle éprouve le goủt d'avoir un dieu, un seul dieu», "elle aurait voulu donner autant qu'elle avait détruit × (p. 213).

On a noté que les romans de femmes reprenaient souvent les schémas des contes de fées. Mais on a été peu attentif à la présence de ces monstres. de ces sorcières. de cette cruauté dans des textes dont on n'a pas voulu entendre la violence. Sans doute était-ce plus rassurant de cantonner la slittérature féminine » dans l'irréel, l'eau de fée, le happy end amoureux.

Dans la Belle Bête. la violence n'est pas seulement dans l'anecdote. dans la logique des actions, de la brutalité aux crimes. Elle est inscrite dans le paroxysme des pulsions, dans les signifiants obsessionnels: «ongles", "griffes», «ricanements», «sorcièren, «démoniaque», «damné», «érocité», a souilluren, "perversion ", "monstren, dans une écriture souvent maladroite, aux phrases coupées à la hache, sèches et délirantes à la fois. L'univers du roman est un univers d' "êtres doubles» habités par des monstres nocturnes. Ces monstres, ce sont les forces inconscientes déchainées, les pulsions qui explosent, les fantasmes destructeurs qui s'emparent de la scène, "grimaçant de monstruosité ‘ (p. 173). On bascule dans l'autre scène où l'on bat. l'on tue. l'on brùle - le frère, la mère, la maison, la terre, soi-même. Soi et l'autre: le démon. Celui qu'on croit être et celui qu'on n'accepte pas d'être. L'écriture devient exhibition d'un grand guignol, miroir grossissant des pulsions. Comme les "enfants * dans le texte jouent et pour eux le jeu est la seule acertitude* pour déjouer une réalité invivable, l'écriture est aussi xjeu», théâtre de l'inconscient et catharsis. Une phrase pourrait être lue comme une mise en abyme du texte: «n exerçant sur l'autre la passion dramatique qui l'étouffait. Faust s'apaisait lui-mème» (p. 196). Le mélodrame est un psychodrame.

Dans les années où fut écrit ce texte, G. Piroué, dans une table ronde sur la "psychologie de la littérature féminine». reprochait au roman de femmes d'être une entreprise de défoulement. Si défoulement il y a, sa mesure est à la violence du refoulement. Et avant tout du refoulé sexuel. 
Dans ce roman. la sensualité est partout, brùlante et pure, bestiale et sauvage. La longue séquence qui décrit, sous le regard de Patrice. l'accouplement féroce et les cris d'amour des chats (p. 116-117) explose dans la mise en scène d'un masochisme "femelle" et du trouble orgastıque de Patrice.

Le texte se construit dans un dialogisme où se combattent et se contaminent le discours de lidéologie chrétienne de la chair comme péché, de la chair comme souillure, et le discours du désir, feu des corps, "corps qui se lève emporté (p. 105). Ce discours contradictoire, inscrit une opposition entre le "pur» et l' «impur ». Impur, l'échange «féroce * des corps de Louise et de Lanz, «vice ignoble», «malsain», véritable cannibalisme: acomme on donne de la chair à manger • (p. 78); impure, I'entrée d'Isabelle au bal, car, dans le roman. le bal de Cendrillon est une danse de sorcière un soir de sabbat : « déchaînée par les flots de son sang», elle dansait a avec fureur ", «torduen; " ses flancs brûlaient $\%$, comme une interdite, dévoilée pour une nuit* (p. 60). Pur, le besoin de contact, de fusion de Michaël et d'Isabelle-Marie, ce jeu « d'amour et de jeunesse «qui permettait tout sans se blesser dans leur chair» (p. 76). ce a mutuel goût d'enfance». Quand le désir de Michaël l'emporte, Isabelle le vit comme une transgression et sanglote: «Elle sentait qu une partie de ces ajeux $\times$ allait prendre fin. Tout serait tellement grave désormais. Tout ressemblerait à Louise. a Lanz, à l'immense tragédie qu'ils déployaient tristement (p. 105). Sexualité impure des adultes qui finit toujours par séparer les amants. Le rêve d'Isabelle est celui qu'expriment tant de romans de femmes: "vivre l'un dans l'autre et continuer les jeux» (p. 133).

Dans un même mouvement, la hantise du péché affronte le désir, la peur de l'échec amoureux mine le besoin de fusion des corps. Dialogısme d'une transgression et dune condamnation d'un "ça déchainé et d'un

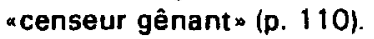

L'être de désir porte en lui son propre opposant, être double, clivé. Dialogisme qu'on pourrait lire comme l'effet sur le désir des femmes du discours dominant sur la sexualité, dans une société profondément marquée par l'influence de l'Eglise.

L'hyperbole du «mal» est bien à la mesure du refoulé: Isabelle brûle parce qu'elle est brûlée.

\section{La difficulté de dire}

Si Goldmann a pu lire ce texte du point de vue des intellectuels révoités mais impuissants, c'est que, dans la révolte contre les mentalités et les valeurs de la communauté traditionnelle, les jeunes intellectuels et les femmes de la nouvelle génération sont du même côté. Et il n'est sans doute pas indifférent que Marie-Claire Blais soit à la fois une intellectuelle et une femme.

Le mal être qui domine le roman, ce pessimisme social, religieux, individuel correspond à une période de transition de la société québécoise. Pour de nouvelles couches - les intellectuels et les femmes - qui restent marginales ou impuissantes dans ces années qui précèdent le réveil québécois. 
la "révolution tranquille" et le surgissement d'un mouvement de femmes, la révolte parait la seule issue sans que soit encore en vue une perspective de véritable changement, de conquète d'identité et de reconnaissance de soi par l'autre.

Crise de valeurs, crise d'identité pour celle (la femme, la communauté) à qui on n'en a jamais reconnu une, déchirée entre des représentations et des aspirations contradictoires, partagée entre le et et le ou: Marie ou Éve. Marie et Eve; canadienne ou française, canadienne et française? Avant que ne tente de se formuler sur le mode de la négation du passé et de l'affirmation d'un a-venir cette nouvelle identité: ni Marie-Ėve, ni canadienne-française mais "femme", mais "québécoisen.

Cette double lecture - possible dans la Belle Bête - resterait encore réductrice si elle ne mettait pas l'accent sur la spécificité de ce texte de fermme qui s'écrit paradoxalement dans le dépouillement et l'outrance, dans une ambivalence qui inscrit un conflit profond, des tensions non surmontées.

Ce qui pourrait être lu dans ce roman, du "point de vue des fernmes", c'est, dans ce temps social èt historique où vivent les femmes dans ce Québec des années 50 qu'il faudrait mieux interroger, la difficulté - commune à tant de femmes de nos sociétés - de dire les femmes sinon dans les images. imposées par l'imaginaire social, occidental ou chrétien, de la prostitution, de la poupée, de la mauvaise mère, de Marie et d'Éve, de Cendrillon et d'Electre, de la belle et de la bête, du bourreau et de la victime, de la victime devenue bourreau; la difficulté de poser les questions de l'identité, du rapport à la mère, à l'autre sexe. la difficulté de dire son désir, de "représenter n ce qui ne parait pas représentable sinon sous la forme du conte ou du mythe, sinon dans la violence et dans l'excès, sous la formë d'un défoulement destructeur: détruire les autres, détruire ses doubles, la Belle, la mère, se détruire, « détruire, dit-elle»; la difficulté d'énoncer même une tentative de réponse sinon dans une purgation cathartique, dans l'ambivalence ou l'antiphrase d'un dénouement: la difficulté de dire ce qui est vécu comme marginal, hors normes sinon dans le décalage, la distance, l'ambivalence de l'énonciation comme pour signifier la dualité et l'impossibilité de s'accepter dans cette dualité, comme pour clamer la révolte et la récuser à la fois.

Goldmann, à un moment où la « nouvelle critique " en France s'enfermait dans le structuralisme linguistique, la clôture du texte et le refus de l'historicité. a tenté de faire une lecture socio-historique des textes. C'est son immense apport.

Mais le socio-historique a une double scène et c'est aussi sur la scène du "privé " que s'écrivent et s'inscrivent les romans et qu'il faut lire les romans de femmes. 
Sur la scène aussı des pulsions, des désirs, des frustrations, des aspirations, du jamais encore dit, du toujours déjá symbolisé dans les figures, archétypes, mythes, scenarii, thèmes romanesques, métaphores, qui ne cessent de se rèver et de se réécrire. S'écrire encore, s'écrire autrement. C'est sur cette scène où les femmes ont été si longtemps cantonnées que prolifèrent dans leurs romans, dans la traversée, le déplacement et la reformulation de l'imaginaire social, un imaginaire - au féminin? - qu'il faut interroger.

Ces scènes sont celles de notre vécu. C'est dans une incessante référence à elles que nous lisons. C'est dans la confrontation du texte et de son questionnement que le critique, lecteur ou lectrice, recourt aux homologies ou aux analogies et construit des «explications * ou encore, dans la fulgurance et le travall d'une "rencontre entre deux inconscients», élabore une interprétation.

Sans doute «le point de vue des femmes * se découvre-t-il d'abord dans l'échange privilégié entre des écrits de femmes et des femmes qui les lisent et s'y lisent.

«Tentative de réponse * qui compenserait «le mélange et les compromis que la réalité impose aux sujets ${ }^{\circ}$, que l'écriture ouvre une a autre scène.. C'est là, sous les masques et les oripeaux des personnages, des stéréotypes culturels, dans les trouvailles formelles, dans les contraintes et les trouées du langage, dans un rythme, une voix, une formule insistante, dans le dialogue du «je» et du $\propto$ on, du «je» et du «elle * que se joue cet autre du réel, autre et réel à la fois, tissé du corps des mots et de la chair du vécu social qu'est. comme tout texte, un roman de femme. C'est là qu'il nous faut tenter de le lire. comme le dit Rimbaud, «littéralement et dans tous les sens», mème si nous faisons, en le déconstruisant et reconstruisant à la fois, que le re-présenter sur les scènes qui sont les nòtres.

1. Lucien Goldmann, *Note sur deux romans de Marie-Claire Blais -. Structures mentales et Création culturelle. Paris, Éditions Anthropos, 1970, p. 401-414.

2. Lucien Goldmann, « La sociologie de la lintérature : situation actuelle et problèmes de méthoden, Revue internationale des sciences sociales. Unesco, $n^{\circ} 4$ (1967), p. 532.

3. L. Althusser, «Soutenance d'Amiens», Positions, Paris, Editions sociales, 1976.

4. Marie-Claire Blais, la Belle Bête, Québec, Institut littéraire du Québec, 1959 : c'est l'édition de référence pour les passages cités.

5. "L'analogon de la ville *, Michel Brùlé, *Introduction à l'univers de Marie-Claire Blais", Sociologie de la littérature. Bruxelles, les Editions de l'institut de sociologie. Université libre de Bruxelles, 1970.

6. Lucien Goldmann, «La méthode structuraliste génétique en histoire de la littérature*, Pour une sociologie du roman. Paris, NRF Gallimard, coll. Idées. 1964, p. 363. 